\title{
Co-existence of Acute Transverse Myelitis and Guillain Barre Syndrome in a Child Following Mumps Infection: A Case Report and Review of Literature
}

\section{Rajniti Prasad*, Pradhap K and Meenakshi Pandey}

Division of Pediatric Neurology, Institute of Medical Sciences, B.H.U., Varanasi, India

*Corresponding Author: Rajniti Prasad, Professor and Chief, Division of Pediatric Neurology, Department of Pediatrics, Institute of Medical Sciences, B.H.U., Varanasi, India.
Received: August 16, 2021

Published: November 10, 2021

(c) All rights are reserved by Rajniti Prasad., et al.

\begin{abstract}
The co-existence of Acute transverse myelitis (ATM) and Guillain-Barre syndrome (GBS) as a result of auto antibodies directed against self-neural antigens following mumps infection in children is rare. We present a 3-years boy admitted with weakness of both lower limbs with provisional diagnosis of transverse myelitis based on clinical and MRI findings. The patient was treated with intravenous methylprednisolone without significant recovery. The diagnosis was later revised to include GBS on the basis of CSF analysis and neurophysiological evidence of acute motor axonal neuropathy. A dual pathology of ATM and GBS may require intensive therapy directed against both diseases.
\end{abstract}

Keywords: Acute Transverse Myelitis; Guillain-Barre Syndrome; Mumps

\section{Introduction}

Acute transverse myelitis (ATM) and Guillain-Barré syndrome (GBS) are immune-mediated processes. ATM comprises a subgroup of noncompressive myelopathies presenting with upper motor neuron signs and is either caused by myelitis due to direct infection of the spinal cord or autoimmune processes [1]. GBS is an inflammatory demyelinating polyneuropathy presenting as acute flaccid lower motor neuron type paralysis characterized by ascending and symmetric weakness of the extremities and hyporeflexia or areflexia, occurring due to antibodies against myelin proteins of peripheral nerves [2]. There are few case reports of GBS and ATM as sequelae of mumps infection [3,4]. We report a 3-years boy who presented concomitantly with ATM and GBS due to mumps infection.

\section{Case Report}

A three years non-immunized male presented with history of swelling in the left side of the cheek for 10 days, fever and breathing difficulty for 3 days, with similar history of cheek swelling in the elder siblings. Child was admitted in a private hospital with di- agnosis of Pneumonia. As the symptoms did not improve, child was referred to our hospital and admitted in PICU. Chest radiograph showed infiltrates, C-reactive protein was raised $(22 \mathrm{mg} / \mathrm{dl})$ and rest of hemogram, Renal function test and liver function test were within normal limits. Child was put on mechanical ventilation and was started on IV antibiotics, fluids and analgesics. Gradually the child condition improved and was extubated and detailed neurological examination was done.

The child had absent deep tendon reflexes and positive Babniski's response. Power of muscle group in the lower limbs was $1 / 5$, while that in upper limbs was $5 / 5$. There was urinary retention, with patchy sensory loss in both lower limbs. MRI brain was normal but of spine done on day 11 of illness, revealed long segment signal intensity changes in the dorsolumbar [D6- L1] spine suggestive of Transverse myelitis [Figure $1 \mathrm{~A}$ and $1 \mathrm{~B}$ ]. Child was given IV Methyl prednisolone at a dose of $30 \mathrm{mg} / \mathrm{kg} /$ day for 5 days.

In hospital stay, child developed progressive weakness of trunk and both upper limbs. On day 15 of the illness, the power in the upper limbs had 2/5. Nerve conduction tests done at day 16 of ill- 


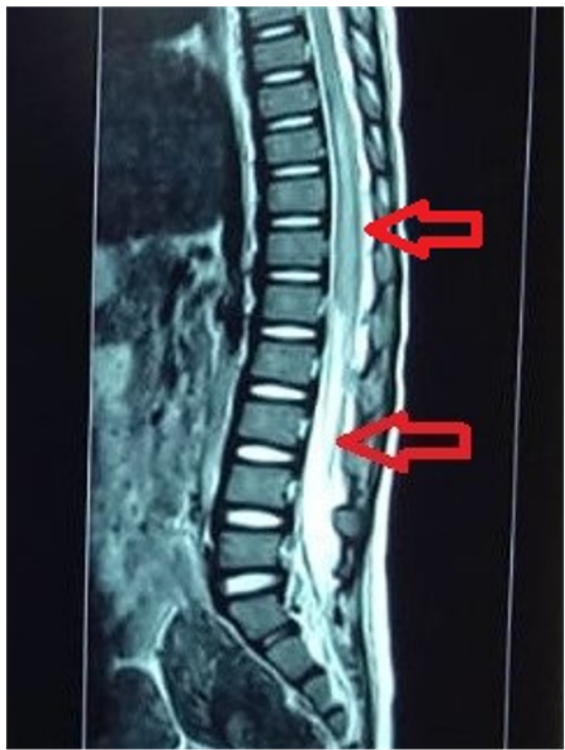

Figure 1A: Sagittal section of MRI spine showing altered signal intensity extending from D6 to L1 level.

ness showed features of motor axonal neuropathy with absence of F-wave in bilateral common peroneal, bilateral sural and ulnar nerves. The right median nerve also had a decreased amplitude and diminished velocity(The amplitude and conduction velocity of less than $3^{\text {rd }}$ percentile are mentioned as abnormal and latency of more than $97^{\text {th }}$ percentile is mentioned as abnormal for sural nerve and ulnar nerve). The sensory conduction was normal [Table 1]. Cerebrospinal fluid (CSF) analysis on day 17 of illness, showed

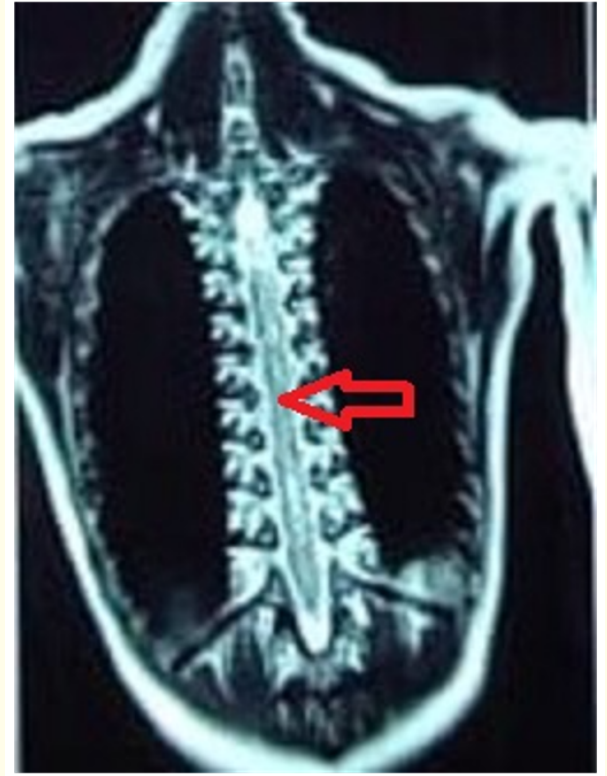

Figure 1B: Coronal section of the MRI spine showing diffuse hyperintensity involving gray matter of spinal cord.

albuninocytological dissociation(cell counts:3 cells $/ \mathrm{mm}^{3,} 100 \%$ lymphocytes, glucose; $65 \mathrm{mg} / \mathrm{dL}$ and protein; $216 \mathrm{mg} / \mathrm{dL}$ ). The nerve conduction studies and CSF findings are suggestive of a pure motor axonal neuropathy, a variant of GBS. The serum IgM Mumps antibody titres were $34 \mathrm{U} / \mathrm{mL}(\mathrm{n}=$ up to $8 \mathrm{U} / \mathrm{mL}$ ), more than four times the normal levels, which confirms the serological evidence of mumps infection. Child was given immunoglobulin $(1 \mathrm{gm} / \mathrm{kg} /$ day intravenously for 2 days). There was no respiratory involvement and the weakness became static after Day 19 of illness.

\begin{tabular}{|c|c|c|c|c|c|c|c|}
\hline & $\begin{array}{c}\text { Latency } \\
(\mathrm{m} / \mathrm{s})\end{array}$ & $\begin{array}{c}\text { Amplitude } \\
(\mathrm{mv} / \mu \mathrm{v})\end{array}$ & $\begin{array}{c}\text { Velocity } \\
\text { (m/s) }\end{array}$ & & $\begin{array}{c}\text { Latency } \\
(\mathrm{m} / \mathrm{s})\end{array}$ & $\begin{array}{c}\text { Amplitude } \\
(\mathrm{mv} / \mu \mathrm{v})\end{array}$ & $\begin{array}{c}\text { Velocity } \\
(\mathrm{m} / \mathrm{s})\end{array}$ \\
\hline \multicolumn{8}{|l|}{ MOTOR } \\
\hline Right median & $2.1 / 4.2$ & $8.6 / 6.8$ & 61.9 & Left median & $-/-$ & - & - \\
\hline Right Ulnar & $-/-$ & - & - & Left ulnar & $-/-$ & - & - \\
\hline $\begin{array}{l}\text { Right Common } \\
\text { peroneal }\end{array}$ & $-/-$ & - & - & $\begin{array}{c}\text { Left common } \\
\text { peroneal }\end{array}$ & $-/-$ & - & - \\
\hline Right Tibial & $-/-$ & - & - & Left tibial & $-/-$ & - & - \\
\hline \multicolumn{8}{|l|}{ SENSORY } \\
\hline Right median & 1.6 & 14.1 & 58.8 & Left median & 1.5 & 19.9 & 54.1 \\
\hline Right Ulnar & 1.5 & 15.9 & 62.5 & Left ulnar & 1.3 & 13.7 & 78.5 \\
\hline F wave & - & - & - & F wave & - & - & - \\
\hline
\end{tabular}

Table 1: Results of nerve conduction studies.

“-”Indicates absence.

*Sural nerves are spared as being sensory and patient had acute motor axonal neuropathy. 
Patient was discharged with advice of physiotherapy. Follow up after 15 days, 1 month and 3 months showed gradual recovery in power of upper and lower limbs. At 3 months, patient was ambulatory with normal power in both upper and lower limbs with normal deep tendon reflexes.

\section{Discussion and Conclusion}

In our patient, there was involvement of both spinal cord and peripheral nervous system. The neurological findings of symmetrical lower limb weakness with patchy sensory loss in both lower limbs, early and persistent bladder involvement, and spinal cord changes in MRI were consistent with ATM. But the lower motor neuron type of weakness, which was initially thought to be due to spinal shock, was later found to be due to GBS, as there was ascending weakness and electrophysiological studies was indicative of a primary peripheral neuropathy and the CSF analysis showing albuminocytological dissociation. With the history of swelling in the cheek prior to this neurological sequelae and similar history of such swelling in the family members, and elevated CSF IgM Mumps antibody levels, diagnosis of mumps leading to concomitant ATM and GBS was made.

Howell., et al. also reported a 14-years- boy with concomitant ATM and GBS, who despite being treated with both intravenous immunoglobulin and high-dose corticosteroids, made a slow and incomplete recovery. They suggested poorer outcome in simultaneous central and peripheral immune-mediated injury, necessitating a combined therapy with immunoglobulin and corticosteroids [5].

Gupta., et al. reported a 4-years boy with concomitant ATM and GBS and might be due to an immune response directed against an antigen that is common to both central and peripheral nervous systems They also recommended aggressive use of steroids along with intravenous immunoglobulin and/or plasmapheresis [6].

Bajaj., et al. reported a case of a young female fulfilling clinical, electrophysiological and neuro-radiological criteria for both ATM and GBS in the context of an acute mumps viraemia predisposing to severe neurological sequelae [7].

Concomitant ATM and GBS should be kept in mind, in patients presenting with weakness, urinary disturbance and areflexia or hyporeflexia. More high-quality evidences are required to understand the pathophysiologic mechanisms underlying this synchronous occurrence to provide early diagnosis and therapeutic approaches.

\section{Bibliography}

1. Krupp LB., et al. "International Pediatric Multiple Sclerosis Study Group criteria for pediatric multiple sclerosis and im- mune-mediated central nervous system demyelinating disorders: revisions to the 2007 definitions". Multiple Sclerosis Journal 19 (2013): 1261-1267.

2. Van den Berg B., et al. "Guillain-Barré syndrome: pathogenesis, diagnosis, treatment and prognosis". Nature Reviews Neurology 10 (2014): 469-482.

3. Duncan S., et al. "Mumps and Guillain- Barre' syndrome". Journal of Neurology, Neurosurgery, and Psychiatry 53 (1990): 709.

4. Venketasubramanian N. "Transverse myelitis following mumps in an adult - a case report with MRI correlation". Acta Neurologica Scandinavica 96 (1997): 328-331.

5. Howell KB., et al. "Concomitant transverse myelitis and acute motor axonal neuropathy in an adolescent". Pediatric Neurology 37.5 (2007): 378-381.

6. Gupta AK., et al. "Coexisting Acute Transverse Myelitis and Guillain-Barré Syndrome in a Child: A Rare Presentation". Journal of Pediatric Neurology 16.4 (2016): 161-163.

7. Bajaj NPS., et al. "Acute transverse myelitis and Guillain-Barre' overlap syndrome with serological evidence for mumps viraemia". Acta Neurologica Scandinavica 104 (2001): 239-242.

\section{Volume 4 Issue 12 December 2021 (C) All rights are reserved by Rajniti Prasad., et al.}

